In this case series of SAH patients that were diagnosed with ruptured blister aneurysms, we investigated the role of highresolution (HR)-MRI Vessel Wall Imaging (VWI) in identifying culprit, ruptured aneurysm diagnoses.

Methods and Materials We performed an IRB-approved retrospective review of patients diagnosed with SAH from 20102019 at our institution. We studied patient demographics, presentations, aneurysm size/locations, initial and follow-up imaging including CT, CTA/MRA, DSA, and MR VWII studies.

Results Seven patients (1 Male: 6 Female, mean age: 52) with ruptured blister aneurysms were identified. Median of HuntHess grade (range: 1-4) and Fisher grade (range: 2-4) at presentation were 1 and 3 respectively. Blister aneurysm were small 1-3 $\mathrm{mm}$ in mean size and broad based neck morphology in the anterior circulation. All but 2 of them were located in non-branching sites. Initial CTA was diagnostic for a ruptured blister aneurysm in 3/7 (43\%), confirmed with DSA. In the remaining 4/7 cases CTA was negative, and DSA identified two of these occult ruptured blister aneurysm initially and two subsequently on 1 week follow-up DSA. HR-MR VWI was performed in $3 / 4$ patients $1-3$ days after presentation and revealed mild to moderate focal enhancement and/or T1 hyperintensity of the small aneurysm wall corresponding to the blister aneurysm sites confirmed by initial or follow-up DSA.

Conclusion HR-MR VWI can provide adjunctive diagnostic information for the identification of occult ruptured blister aneurysm in SAH patients, when combined with conventional imaging studies such as CTA and DSA. HR-MR VWI may assist in accelerate the time to definite diagnosis in cases with negative initial CTA or DSA findings.

Disclosures Y. Moazeni: None. R. Abdalla: None. M. Sukumaran: None. D. Cantrell: None. A. Shaibani: None. M. Hurley: None. B. Jahromi: None. M. Potts: None. S. Ansari: None.

\section{E-186 THE WOVEN ENDOBRIDGE DEVICE FOR RUPTURED INTRACRANIAL ANEURYSMS: A SYSTEMATIC REVIEW AND META-ANALYSIS}

N Alotaibi*, P Harker, F Robertson, J Vranic, M Koch, C Stapleton, T Leslie-Mazwi, A Patel. Neurosurgery, Massachusetts General Hospital, BOSTON, MA

\subsection{6/neurintsurg-2020-SNIS.217}

Background Compared to stent-assisted coiling the Woven EndoBridge (WEB) device offers the advantage of treatment of wide-necked aneurysms without antiplatelet medications. However, the safety and efficacy of WEB is yet to be fully defined in the setting of aneurysmal subarachnoid hemorrhage $(\mathrm{aSAH})$, given concerns regarding the delayed nature of aneurysm thrombosis.

Methods The search protocol was developed a priori according to the Preferred Reporting Items for Systematic Reviews and Meta-Analyses (PRISMA) guidelines. A literature search using the OVID Medline, Embase, Web of Science, and Cochrane Library databases from inception to February 2020 was performed using relevant key words to identify cases of aSAH that were treated with WEB. The primary outcome measure was radiographic complete occlusion rates. Secondary outcomes included rebleeding and complications rates. All pooled event rates were calculated using random effect model.
Results Six articles, all of which were observational case series, representing 261 ruptured aneurysms were included for analysis (average follow up time was 4 months). Most of these aneurysms were in the anterior circulation (>60\%). The likelihood of a complete radiographic occlusion following WEB for ruptured aneurysms was $66.6 \%$ (95\% CI $47-$ $74 \%$ ). Rebleeding rates were $2.8 \%$ (95\% CI 1-6\%). The pooled event rate for complications (procedural aneurysm rupture, thromboembolic, and device protrusion in the parent artery) across five studies was $17.9 \%$ (95\% CI 9-32\%). Overall retreatment rates were 10\% (95\% CI 4-22\%) and the need for early or delayed stent placement was 5\% (95\% CI 1-12\%). There was no significant heterogeneity between studies in rebleeding and stent placement outcomes $\left(I^{2}<25 \%\right)$.

Conclusion The WEB device in aSAH appears to provide similar protection against rebleeding and retreatment rates that are comparable to traditional approaches; however, there is a higher rate of incomplete radiographic occlusion and operative complications. Long-term prospective studies are needed to fully delineate the role of WEB embolization in aSAH.

Disclosures N. Alotaibi: None. P. Harker: None. F. Robertson: None. J. Vranic: None. M. Koch: None. C. Stapleton: None. T. Leslie-Mazwi: None. A. Patel: None.

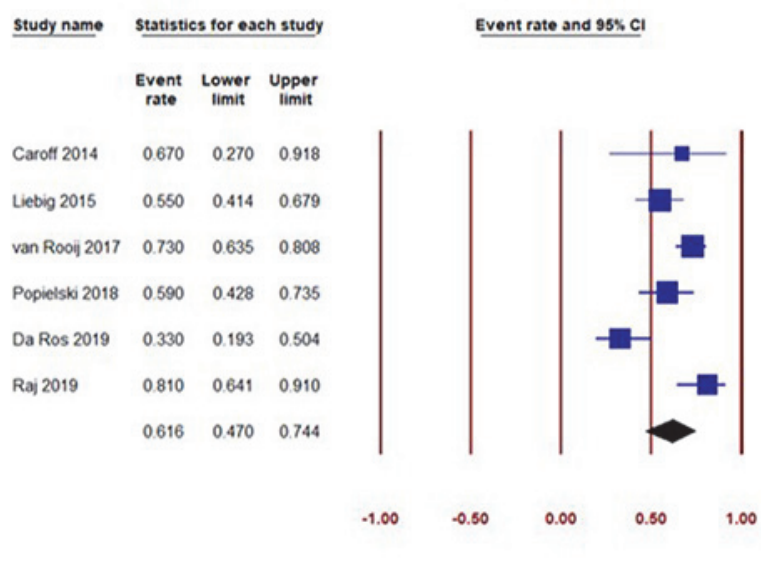

Complete occlusion at 3-6 months follow up (12: $76 \%)$

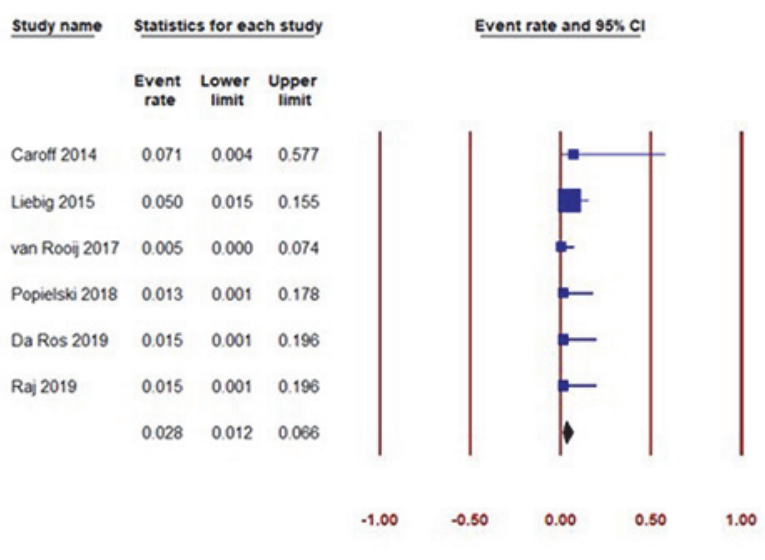

Rebleeding rates at last follow up $\left(\mathrm{I}^{2}: 0 \%\right)$

Abstract E-186 Figure 1 